\title{
Lévitation magnétique, une approche objet-projet
}

\author{
Lotfi BAGHLI, Abderrezak REZZOUG \\ Lotfi.Baghli@green.uhp-nancy.fr \\ Nancy Université, Faculté des Sciences, BP 239, 54500 Vandoeuvre
}

\begin{abstract}
RESUME Cet article présente l'essentiel de l'étude que l'on peut mener autour d'un objet-projet. Il s'agit de la lévitation magnétique. Nous proposons la réalisation expérimentale, la modélisation et la simulation d'un tel système. Le public visé est de niveau Master. L'intérêt de cette approche est de motiver l'étudiant tout en lui transmettant des enseignements autour de différentes matières : Automatique (modélisation, identification), circuits magnétiques, microcontrôleur (ADC, PWM, UART), électronique de puissance, langage C, MATLAB ${ }^{\circledR}$. La présentation de l'étude se prolonge à travers notre site web pour y télécharger librement les schémas électroniques et les programmes réalisées.
\end{abstract}

Mots clés : Lévitation, FEMM, dsPIC, microcontrôleur, MATLAB ${ }^{\circledR}$.

\section{INTRODUCTION}

Le but de cet article est de présenter un exemple d'approche d'enseignement autour d'objet ludique. Il s'agit de la lévitation magnétique d'objets collés à un aimant. Le système de lévitation permet, grâce à une régulation numérique, de contrôler la distance de lévitation. Ce projet permet d'aborder plusieurs thèmes connexes que l'on retrouve en enseignement des sciences pour l'ingénieur. On montre l'interaction et le lien entres différentes disciplines et qu'une base générale de connaissances, suffisamment large, est nécessaire pour pouvoir surmonter les différentes difficultés liées à un tel projet.

La théorie montre que ce système est non linéaire et difficile à modéliser. De plus, il est naturellement instable. On peut le réaliser de manière analogique [1][2] ou numérique et le régler par tâtonnement. On peut également chercher une modélisation qui reste à la portée d'un étudiant de Master (1 ou 2).

Nous présentons une approche de modélisation qui permet le calcul de l'induction magnétique et des forces par un logiciel libre d'élément fini, une identification des courbes obtenues conduit à un modèle valide autour d'un point de fonctionnement. Cette approche permet de simuler le système voire même de régler les paramètres du correcteur.

La réalisation expérimentale du projet est présentée et donne lieu à des discussions quant aux aspects que l'on peut greffer au projet initial, options que l'on pourra étudier en M2 à la suite d'une première approche en M1 par exemple.

\section{DESCRIPTION DE L'OBJET D'ETUDE}

L'objet de notre étude se compose d'une bobine à noyau de fer constitué par un boulon ferromagnétique. Ce dernier maintient la bobine au support. Elle comporte n spires parcourues par un courant I. En dessous du noyau, à une distance de $1 \mathrm{~mm}$, on place une sonde à effet Hall qui permet de mesurer la valeur de l'induction magnétique $B$ de manière linéaire (Honeywell SS459). Cette bobine attire un aimant
(NFeB) en dessous duquel on peut coller un objet (stylo par exemple). La fonction de ce système est de réaliser la lévitation de l'objet (Fig 1). Le courant dans la bobine est régulé par un microcontrôleur et appliqué par un hacheur 4-quadrants de manière à ce que, si l'objet (et donc l'aimant) s'éloigne de la sonde à effet Hall, alors le courant $I$ devient négatif et l'aimant est attiré. Au contraire, si l'aimant s'approche trop, l'induction au niveau de la sonde magnétique s'inverse et la régulation imposera un courant positif qui fera s'éloigner l'aimant.

Le processus de lévitation magnétique est naturellement instable et il convient d'apporter une régulation rapide pour maintenir en équilibre l'ensemble aimant - objet. On peut obtenir des distances de lévitation de l'ordre du centimètre.

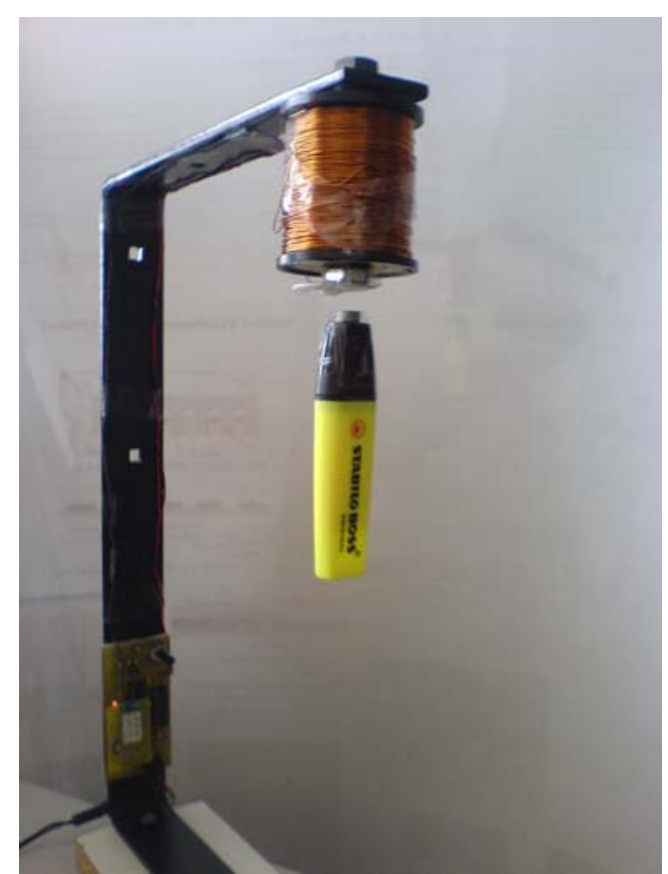

Fig 1. Maquette expérimentale, lévitation d'un marqueur accroché à un aimant. 
La lévitation de petits objets a donné lieu à des réalisations ludiques, que l'on retrouve sur quelques sites web [3][4], avec une régulation analogique.

Nous avons choisi une approche numérique qui ne réduit pas forcément, pour cette application, le nombre de composants utilisés, mais qui offre une grande souplesse d'adaptation du système à l'étude que nous voulons en faire.

Cela permet entre autres le changement en ligne les paramètres du régulateur, la transmission des informations et le pilotage à distance (PC, PocketPC ou téléphone Java compatible MIDP) via une liaison Bluetooth $^{\circledR}$. Cela permet d'envisager de nombreuses fonctionnalités qu'offre un système numérique à microcontrôleur (ADC, PWM, interruption, UART).

\section{MODELISATION DU SYSTEME}

La modélisation d'un tel système est difficile car le problème électromagnétique est du type "ouvert". C'est-à-dire que les lignes de champs se referment dans l'air. Le calcul analytique de la force $F_{z}$ qui agit sur l'aimant n'étant pas simple, nous avons opté pour un calcul numérique par éléments finis. Nous avons choisi le logiciel libre FEMM [5] que nous avons déjà utilisé pour d'autres applications pédagogiques [6].

Comme pour toute étude, nous devons faire des hypothèses simplificatrices :

On ne tient pas compte de la saturation magnétique, c'est-à-dire que le matériau ferromagnétique du noyau est linéaire et ne sature pas. Cela reste vrai vu le niveau de l'induction $B$ puisque le circuit magnétique est ouvert. Cette hypothèse de travail réduit considérablement le temps de calcul requis par le logiciel FEMM.

Le terme $\frac{\partial A_{z}}{\partial t}$ est nul ( $\underline{A}$ étant le potentiel magnétique) : nous considérons que le problème électromagnétique est du type magnétostatique, afin que le logiciel puisse le résoudre. Cela suppose que nous négligeons la constante de temps électrique devant celle mécanique du système.

Le logiciel permet de rentrer des géométries à structure axiale en coordonnées cylindriques $(r, z)$, ce qui est le cas de notre système (bobine + aimant) (Fig 2).

On lance le calcul puis on affiche $B_{z}$ le long de l'axe $r=0$ (Fig 3). On s'intéresse à la valeur de l'induction à une distance de $1 \mathrm{~mm}$ en dessous de la bobine. On appelle cette valeur $B_{z 1}$ (à $z=-1 \mathrm{~mm}$ ). C'est à cet endroit que l'on place la sonde à effet Hall dans le système réel.

On cherche également la force $F_{z}$ exercée sur l'aimant. Le logiciel FEMM la calcule à l'aide du tenseur de Maxwell [7].

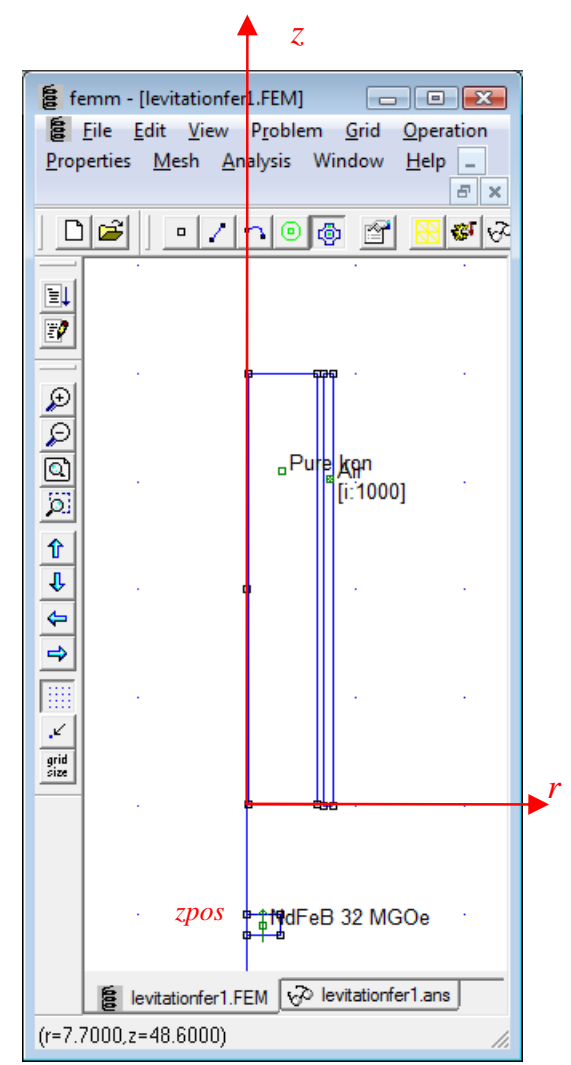

Fig 2. Géométrie du système rentrée dans

le logiciel de calcul de champ.

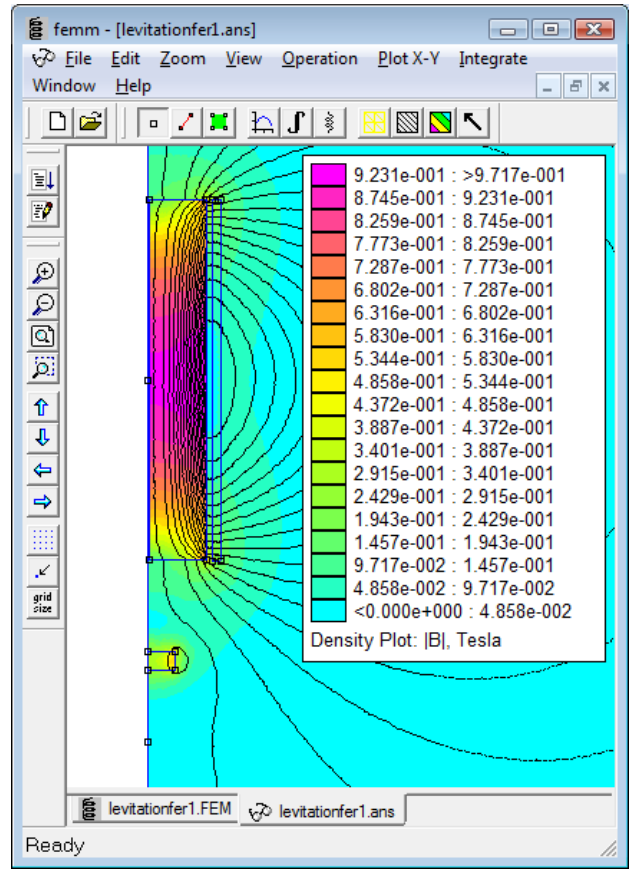

Fig 3. Résultats d'un calcul de champ.

Il existe une extension de FEMM fort intéressante qui permet de piloter le logiciel à l'aide d'un logiciel de calcul comme Matlab ${ }^{\circledR}$ ou Octave. Nous utilisons cette fonctionnalité pour faire varier automatiquement la 
position zpos de l'aimant entre 0 et $-10.5 \mathrm{~mm}$ ainsi que le courant $I$ de la bobine de $-2.5 \mathrm{~A}$ à $2.5 \mathrm{~A}$. Ainsi, nous avons balayé $15 x 5$ configurations de calcul.

Les figures Fig 4 et Fig 5 montrent la variation de $B_{z 1}$ et $F_{z}$ en fonction de la position de l'aimant pour différentes valeurs de courant.

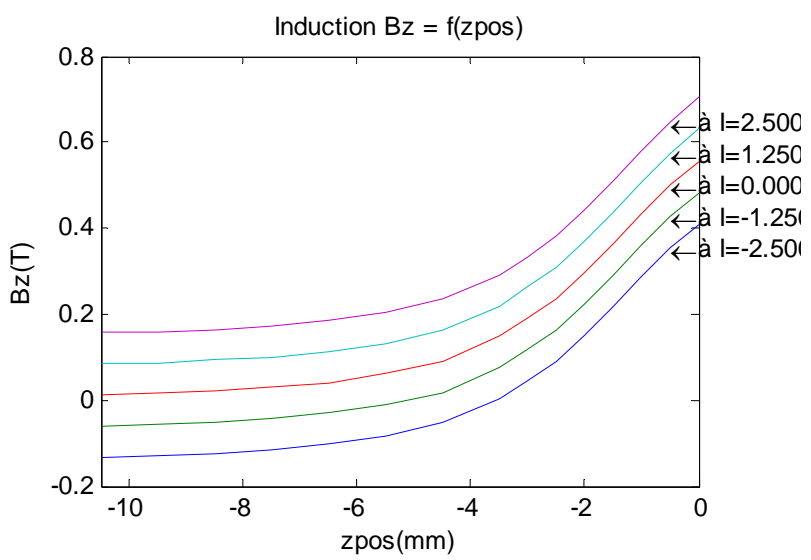

Fig 4. Variation de $B_{z 1}$ en fonction de la position de l'aimant pour différentes valeurs de courant.

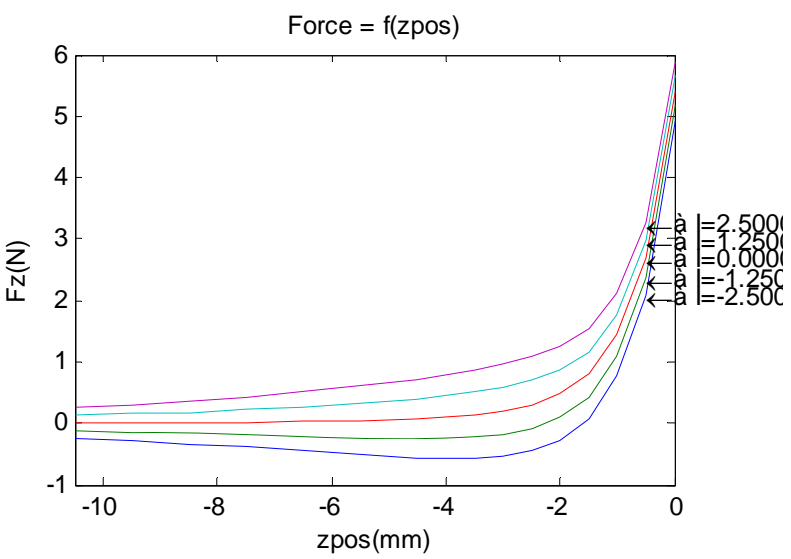

Fig 5. Variation de $F_{z}$ en fonction de la position de l'aimant pour différentes valeurs de courant.

Ces courbes ne sont pas continues mais discrètes aux points de calcul. Si nous voulons simuler le fonctionnement du système nous devons approximer ces relevés par des fonctions polynômiales ou par des fractions de polynômes sur le domaine de l'étude.

Les courbes $B_{z 1}$ ont été approximées de manières indépendantes suivant zpos et $I$. En effet, l'écart entre les courbes, quand le courant varie, reste constant (Fig 4 et Fig 6). La fonction résultante est :

$$
\begin{aligned}
B_{z 1}(\text { zpos, } I)= & \frac{\mathrm{p} 1 \cdot z p o s+\mathrm{p} 2}{z_{p o s}^{2}+\mathrm{q} 1 \cdot z p o s+\mathrm{q} 2} \\
& +\mathrm{Ip} 1 \cdot I+\mathrm{Ip} 2-B_{z 1 \text { fffset }}
\end{aligned}
$$

Avec les coefficients identifiés suivant la méthode des moindres carrés.

Ip1 = 0.05894;

Ip2 = 0.5578; $\mathrm{p} 1=0.3136 ;$

p2 = 4.282;

q1 =-0.3873;

q2 = 7.712;

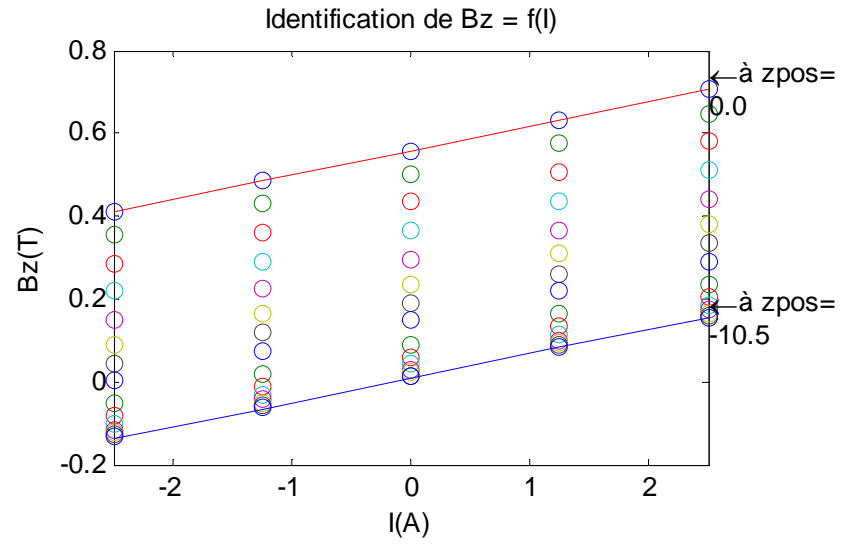

Fig 6. Variation de $B_{z 1}$ en fonction du courant pour différentes positions de l'aimant.

Nous remarquons une bonne concordance de l'identification (Fig 7 et Fig 8) avec le relevé des calculs par éléments finis.

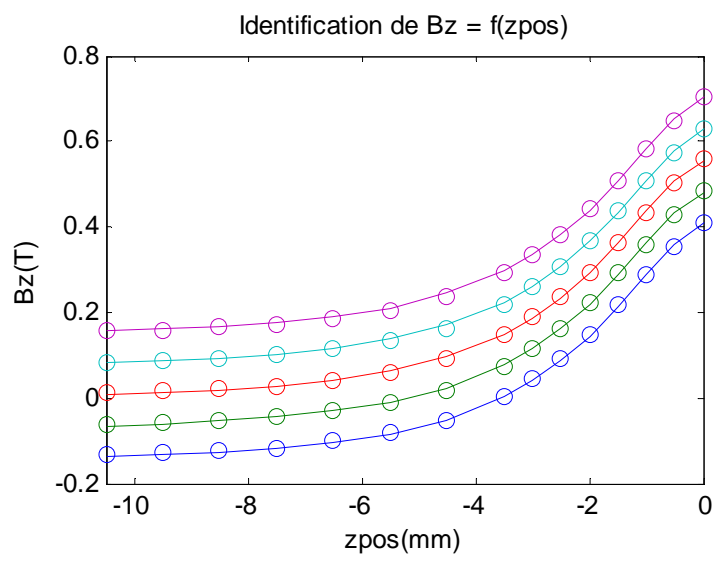

Fig 7. Identification de $B_{z 1}$ sur le domaine de l'étude.

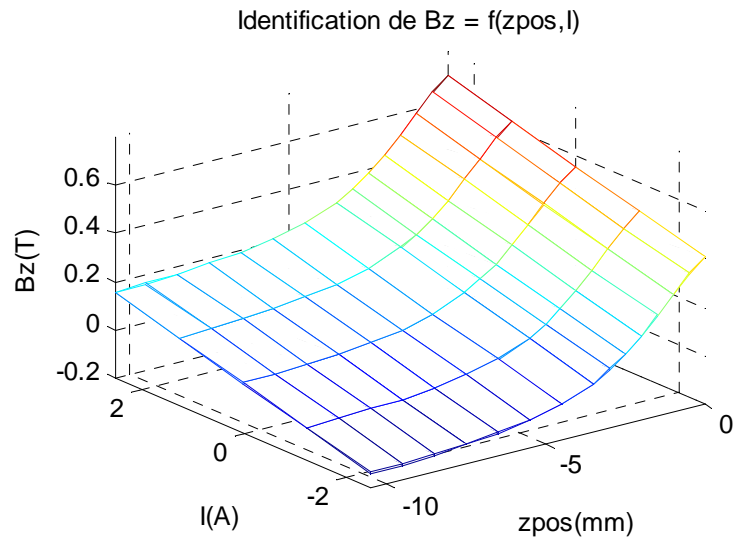

Fig 8. Identification de $B_{z 1}$ sur le domaine de l'étude (3D), les deux surfaces se superposent. 
Concernant la force verticale, le problème est plus compliqué car l'écart entre les courbes (Fig 5) varie également avec la position. Nous traçons (Fig 9) la différence entre les forces extrêmes (à -2.5A et 2.5A).

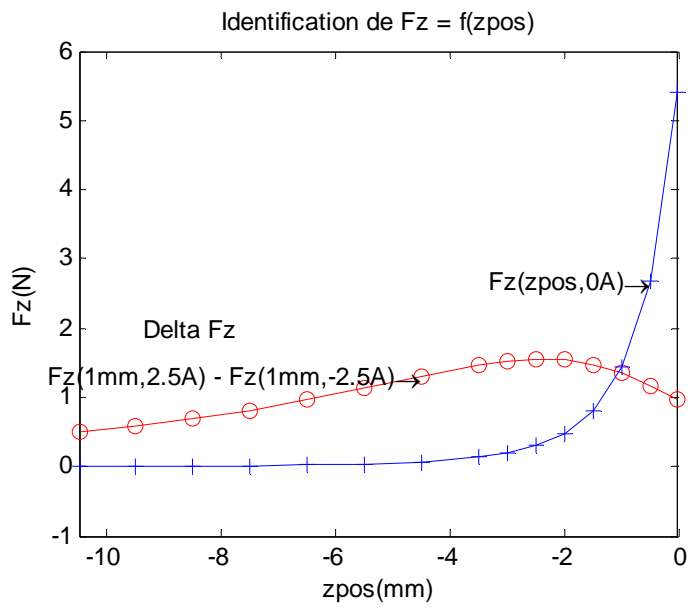

Fig 9. Ecart entre les forces extrêmes de $F_{z 1}$ en fonction de la surface d'état.

L'identification de cet écart aboutit à un polynôme du $4^{\text {ème }}$ ordre de coefficients FIp1px.

Nous adoptons ensuite les mêmes méthodes d'identification que précédemment, avec un ordre plus élevé pour le dénominateur :

Fp1 =-0.2948;

Fp2 =-11.23;

$\mathrm{Fq1}=-1.396$;

$\mathrm{Fq} 2=3.174$

Fq3 =-2.075;

et

FIp1 $=0.1942 ; \%$ FIp1 est à remplacer par le

FIp2 = 5.413; \% polynôme du 4ème ordre

La fonction résultante est :

$$
\begin{aligned}
F_{z}(\text { zpos, } I) & =\frac{\mathrm{Fp} 1 \cdot z p o s+\mathrm{Fp} 2}{\text { zpos }^{3}+\mathrm{Fq} 1 \cdot \mathrm{zpos}^{2}+\mathrm{Fq} 2 \cdot \mathrm{zpos}^{+\mathrm{Fq} 3}} \\
& +\left(\begin{array}{l}
\mathrm{FIp} 1 \mathrm{p} 1 \cdot \mathrm{zpos}^{4}+\mathrm{FIp} 1 \mathrm{p} 2 \cdot \mathrm{zpos}^{3} \\
+\mathrm{FIp} 1 \mathrm{p} 3 \cdot \mathrm{zpos}^{2}+\mathrm{FIp} 1 \mathrm{p} 4 \cdot \mathrm{zpos}^{2} \mathrm{FIp} 1 \mathrm{p} 5
\end{array}\right) \cdot I \\
& +\mathrm{FIp} 2-B_{z 1 \mathrm{ofset}}
\end{aligned}
$$

L'identification est correcte et a également pu se faire facilement avec les fonctions de Matlab ${ }^{\circledR}$. Cela est reflété par les courbes de Fig 10 et Fig 11.

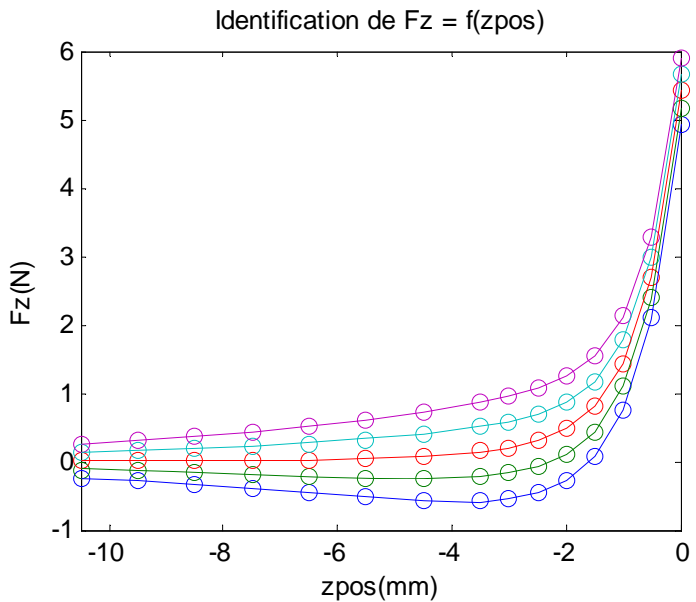

Fig 10. Identification de $F_{z}$ sur le domaine de l'étude.

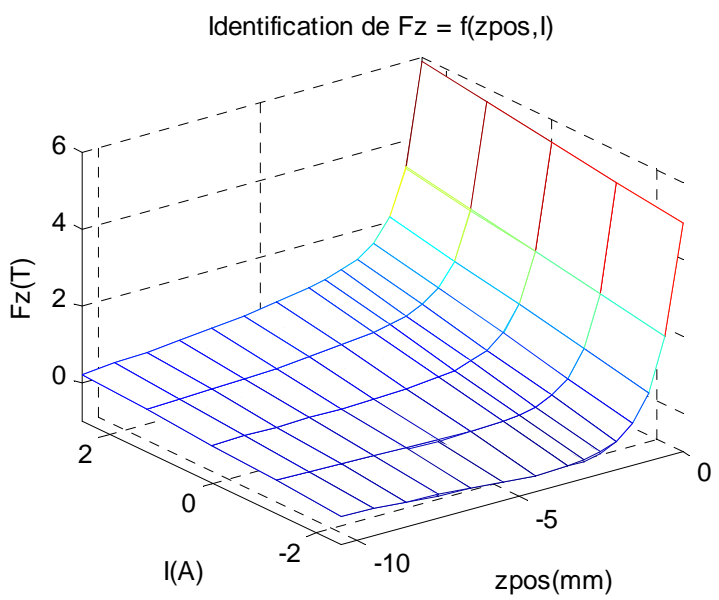

Fig 11. Identification de $F_{z}$ sur le domaine de l'étude (3D), les deux surfaces se superposent.

\section{SIMULATION}

L'intérêt d'avoir des fonctions $B_{z 1}$ (zpos, $I$ ) et $F_{z}$ (zpos, $I$ ) continues en tout point et non pas des fonctions discrètes, est de permettre la simulation temporelle du système.

Le système est naturellement instable. Si on lâche l'aimant d'une position donnée et en fonction de la valeur du courant dans la bobine, il va se coller au noyau de la bobine ou alors tomber. Nous avons limité sa course dans la simulation au domaine de validité de l'étude $(-10.5 \mathrm{~mm} \leq$ zpos $\leq 0.1 \mathrm{~mm})$.

\subsection{Sans la régulation}

En boucle ouverte, nous imposons un courant fixe $I=1.4$ A et relâchons l'aimant de zpos $=3.75 \mathrm{~mm}$ : 

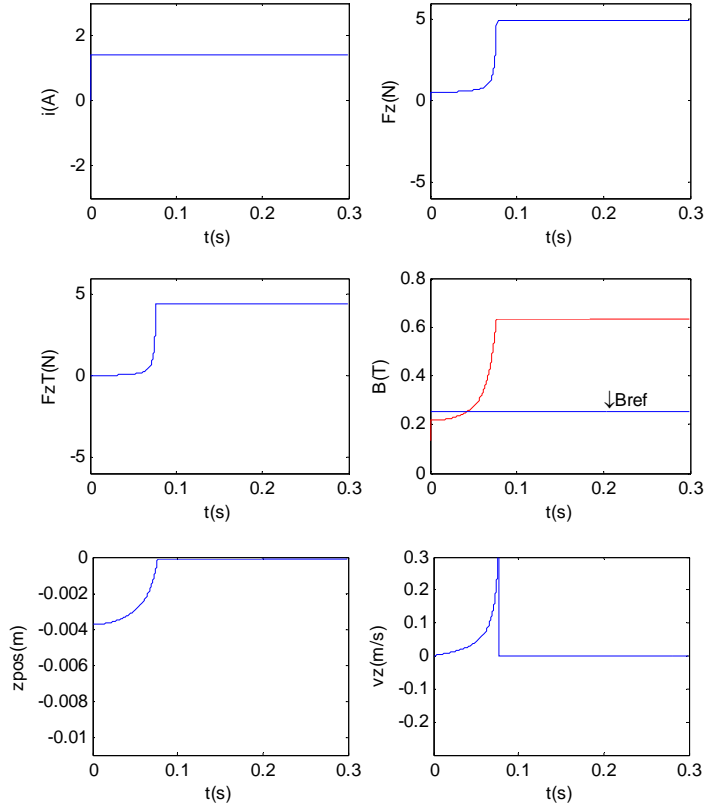

Fig 12. Résultats de simulation en boucle ouverte

$$
(I=1.4 \text { A et } z p o s 0=3.75 \mathrm{~mm}) \text {. }
$$

La force totale $F_{z T}$ comprend, en plus de $F_{z}$, le poids de l'objet et la force de frottement. Elle doit être nulle pour que l'objet soit en équilibre.

La courbe bleue $B_{\text {ref }}$ représente l'induction de référence autour de laquelle nous procéderons par la suite à la régulation.

Nous imposons également un courant fixe $I=1.3$ A et relâchons l'aimant de zpos $=3.75 \mathrm{~mm}$ :
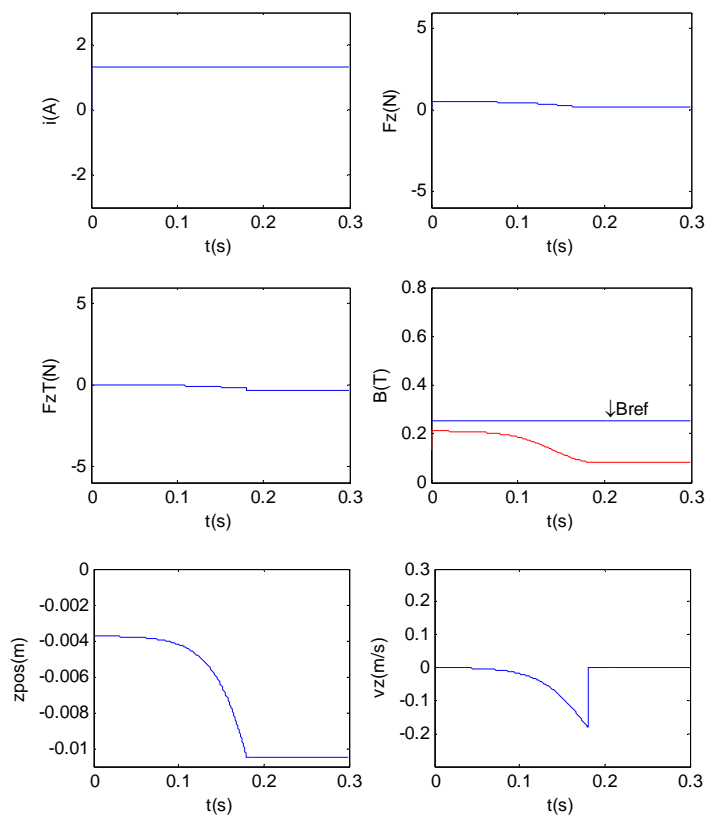

Fig 13. Résultats de simulation en boucle ouverte

$$
(I=1.3 \text { A et } z p o s 0=3.75 \mathrm{~mm}) .
$$

Nous remarquons dans les deux cas que le système va rapidement en buté. Il colle au noyau pour $I=1.4 \mathrm{~A}$ (Fig 12) et tombe pour I=1.3 A (Fig 13).

\subsection{Avec la régulation}

Nous introduisons une boucle de régulation suivant le schéma synoptique de la Fig 14.

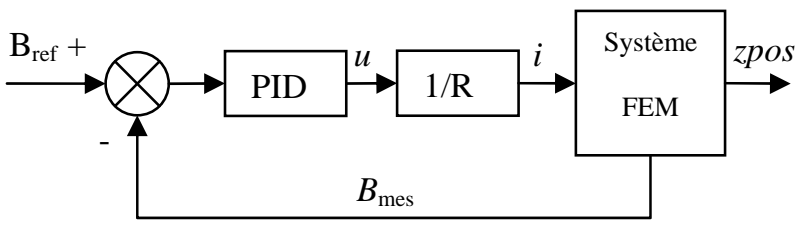

Fig 14. Schéma synoptique de la régulation du système.

On remarque (Fig 15) que le système oscille autour d'une position d'équilibre. Il faudra augmenter le facteur d'amortissement du système pour le stabiliser d'avantage. Pour cela, on pourra rajouter un circuit électriquement fermé (un écrou ou une rondelle) à proximité de l'aimant afin que les variations de champ induisent des courants qui produiront une force tendant à s'opposer au mouvement.
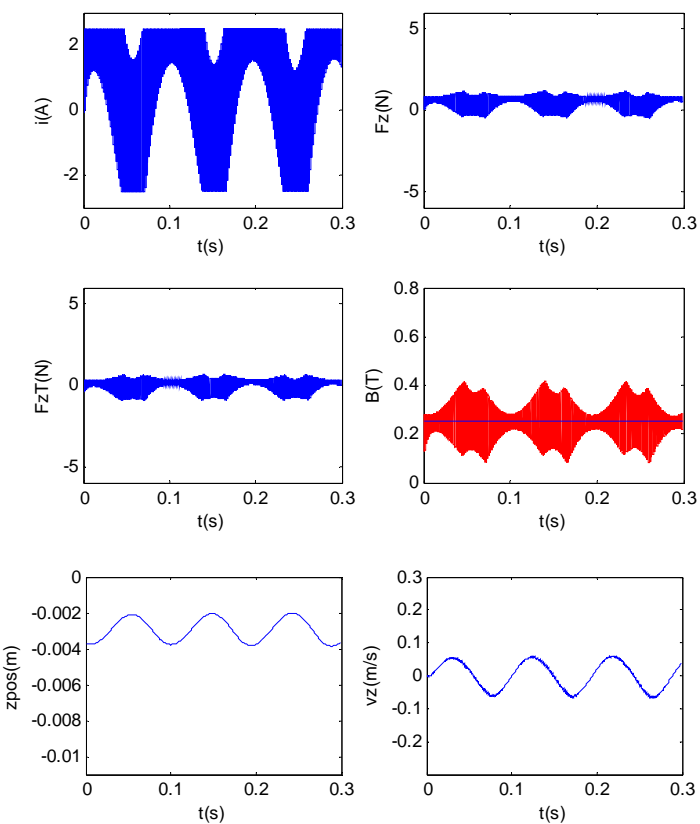

Fig 15. Résultats de simulation en boucle fermée

$(z p o s 0=3.75 \mathrm{~mm})$.

\section{REALISATION}

La commande du système est implantée sur un microcontrôleur dsPIC 30F3010 mais peut l'être sur n'importe quel contrôleur numérique disposant d'entrées analogiques (CAN ou ADC) et de sorties MLI. Il doit en effet quantifier la référence $B_{\text {ref }}$ mais aussi la mesure de l'induction $B_{\text {mes }}$ fournie par la sonde à effet Hall. 
Une approche de réalisation simplifiée (sans utiliser de microcontrôleur) a été effectuée par Marsden [3].

L'intérêt de notre approche est de donner la possibilité d'intervenir sur la valeur de référence de l'induction ainsi que sur les paramètres du régulateur.

La carte électronique comporte principalement le microcontrôleur dsPIC et l'onduleur triphasé L6234. On a choisit ce composant au lieu d'un hacheur 4quadrants (L298 ou autre) à cause de sa compacité et de l'intégration des DRL (diodes de roue libre). Il permet de fournir jusqu'à $2.5 \mathrm{~A}$ pour les faibles tensions d'alimentation compatibles avec notre cahier des charges.

Seuls deux bras de l'onduleur sont utilisés. On ne dispose, pour ce convertisseur, que de la commande des MOSFET hauts. La commande des MOSFET bas se fait directement en interne de manière complémentaire. Afin d'avoir un fonctionnement en hacheur 4-quadrants, les 2 commandes que l'on envoie aux bras de l'onduleur sont donc complémentaires.

Sur la carte, nous avons disposé un connecteur SIL5 permettant de brancher le module de programmation via l'interface ICSP (In Circuit Serial Programming). Il sert à reprogrammer le dsPIC sans avoir à l'enlever de la carte. Il permet également de procéder au débogage du programme via MPLAB ${ }^{\circledR}$ (environnement intégré de développement, IDE de Microchip).

A ce propos, nous recommandons d'utiliser le Pickit 2 au lieu de l'ICD2 comme module de programmation et de débogage. En effet, il coûte 6 fois moins cher pour les mêmes fonctionnalités. Il a de plus un connecteur qui est déjà au format SIL au lieu de la contraignante fiche RJ11 de l'ICD2. Les membres de la famille PIC et dsPIC, que le Pickit 2 permet de programmer, s'est largement étoffée et couvre un très grand nombre de cibles ${ }^{1}$.

Nous avons rajouté un connecteur SIL4 permettant de relier le dsPIC via ses pattes UART RX/TX à tout système de communication série à nivaux logiques $(0$ / $3 \mathrm{~V})$. Nous y avons connecté un module Bluetooth ${ }^{\circledR}$

1 Target : microcontrôleur cible sur lequel le programme sera flashé.
RN41 de chez Roving Networks, disposant du profil SPP dans sa pile logicielle. Il permet de remplacer la liaison série filaire entre 2 ports COM distants de moins de $10 \mathrm{~m}$. Ainsi nous pouvons envoyez et recevoir les informations à partir d'un PC, PocketPC ou téléphone portable disposant de la liaison Bluetooth ${ }^{\circledR}$.

Concernant le programme de commande que nous avons développé pour cette application et qui est implanté sur le dsPIC à l'aide des outils sus-cités, il commence tout d'abord par configurer les ports d'entrées sortie et la liaison UART puis il installe une MLI avec un timer à $16 \mathrm{kHz}$ qui va lancer l'ADC (convertisseur analogique-numérique) à chaque fois que le timer atteint sa période de comptage. Le programme installe une routine de service d'interruption (ISR)_ADCInterrupt qui est appelée automatiquement sur un évènement EOC (End Of Conversion) de l'ADC. Cela permet de gagner beaucoup de temps de calcul, puisque le microcontrôleur n'est jamais bloqué à attendre l'instant de lancement de la conversion ADC ou la fin de la conversion.

C'est dans la routine de traitement de l'interruption que toutes les tâches synchrones sont traitées. On commence par lire les buffers qui ont été attribuées aux entrées ADC du dsPIC puis on exécute la routine du régulateur PID du système présenté en Fig 14. On rafraîchit alors les registres de comparaison des sorties MLI.

L'envoi, la réception et le décodage des ordres via l'UART sont des fonctions qui s'exécutent avec une priorité moindre que celle de l'ISR de manière à ne pas perturber l'acquisition de données et la régulation.

La description de la réalisation ainsi que les schémas électroniques sont disponibles sur la page web d'accompagnement de cet article [8].

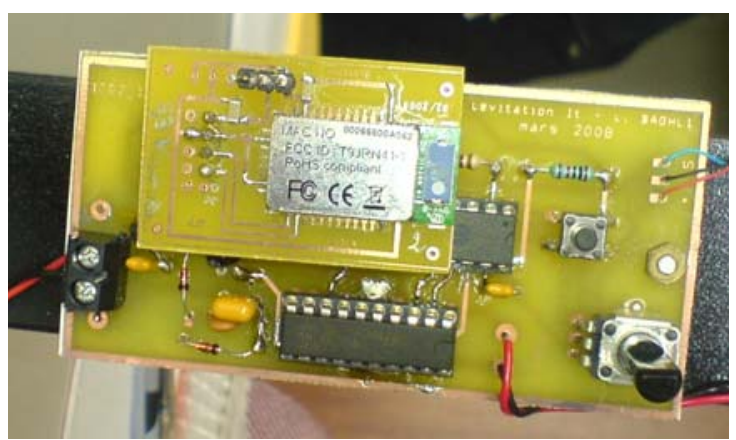

Fig 16. Maquette expérimentale, détail de la carte de commande et du circuit Bluetooth ${ }^{\circledR}$.

\section{INTERFACE HOMME MACHINE}

Nous avons réalisé trois interfaces de contrôle à distance : une Midlet Java pour téléphone mobile 
MIDP2, une interface sur PocketPC et une interface sous Windows ${ }^{\circledR}[8]$.

La Midlet Java permet la commande du système via le dsPIC et le module Bluetooth ${ }^{\circledR}$. On peut changer les paramètres du régulateur PID numérique à distance mais également donner l'ordre de sauver les valeurs dans la EEPROM du dsPIC. Ces paramètres seront alors réutilisés automatiquement à la prochaine remise sous tension du système dsPIC, le rendant ainsi indépendant de l'outil de programmation (ICD 2).

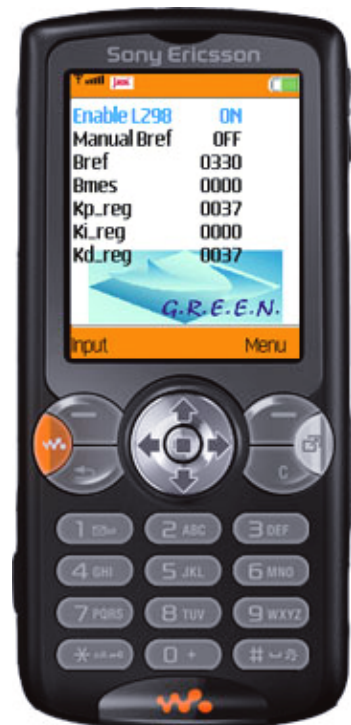

Fig 17. Midlet Java de l'interface de contrôle BT.

Les versions pour PocketPC et pour Windows ${ }^{\circledR}$ permettent également de commander le dsPIC par liaison Bluetooth ${ }^{\circledR}$. En outre, elles offrent la possibilité de communiquer dans les deux sens et de rapatrier la mesure instantanée $B_{\text {mes }}$, c'est-à-dire $B_{z 1}$.

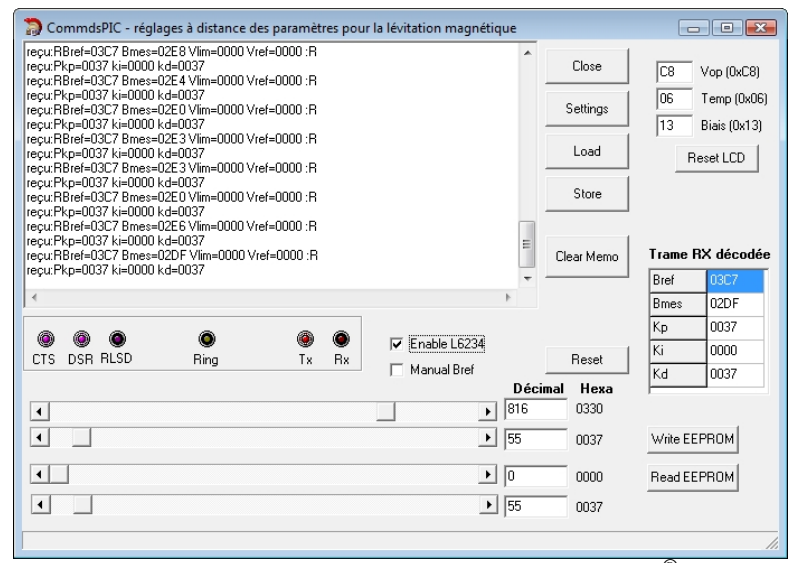

Fig 18. Interface de contrôle sous Windows ${ }^{\circledR}$.

\section{CONCLUSION}

Cette étude présente les possibilités qu'offre le sujet de la lévitation magnétique à des fins pédagogiques pour des projets tutorés, par exemple. On peut étudier différents sujets dans le domaine des sciences pour l'ingénieur. La plus grande difficulté concerne la modélisation du système à des fins de simulation. La réalisation de la maquette expérimentale est aisée.

Nous espérons que ce projet, diffusé sur internet et à travers cet article, se trouvera copié et dupliqué. Nous serions heureux d'avoir des retours et d'éventuelles améliorations à la présente étude et réalisation.

\section{Remerciements}

Les auteurs tiennent à remercier les personnes suivantes pour l'aide qu'elles ont apportée à la réalisation des pièces mécaniques et des cartes électroniques :

J.F. Pautex, ATELA - UHP.

J. Zins, AIP - UHP.

\section{Bibliographie}

[1] K. H. Lundberg, K. A. Lilienkamp, G. Marsden, " Low-Cost Magnetic Levitation Project Kits", IEEE Control Systems Magazine, October 2004 issue. [En ligne].

Disponible: http://www.arttec.net/Press/IEEE/LMarsden.pdf

[2] R. Hoadley, "Experiments with magnetic levitation", [En ligne]. Disponible : http://my.execpc.com/ rhoadley/magsus.htm

[3] Guy Marsden, "Levitation! Float objects in a servo-controlled magnetic field", Nuts \& Volts Magazine, September 2003 issue. [En ligne]. Disponible:

http://www.arttec.net/Press/N\&V/Levitation.pdf

[4] Guy Marsden, "magnetic levitation kit", [En ligne]. Disponible: http://www.arttec.net/Levitation/Gallery/Levitation_Ap plications.htm

[5] D. C. Meeker, Finite Element Method Magnetics, Version 4.0.1 (03Dec2006 Build), [En ligne]. Disponible : http://femm.foster-miller.net

[6] L. Baghli, A. Rezzoug, "Actionneurs linéaires : MRVlin et MSlin, un projet pédagogique", J3eA, Vol. 7 No. Hors Série 1 (février 2008), Special Edition: CETSIS 2007, (http://www.j3ea.org/) 16381963.

[7] M. Jufer, Traité d'électricité vol IX. Electromécanique, Ed. PPURomandes, 3ème édition, 1995, 384p.

[8] L. Baghli, "Lévitation magnétique", [En ligne]. Disponible: http://www.baghli.com/dspic_levitation.php 